Result. Key findings included:

High rates of physical comorbidities among psychiatric inpatients of all ages

Novel illnesses occurring during admissions

Evidence that patients are not receiving adequate physical healthcare from wider NHS

Junior doctors receiving inadequate support from Seniors and acute Hospital services when managing physical illnesses

Poor recording of cardiometabolic monitoring with few interventions delivered (even when indicated) and challenges finding relevant data in records.

During the MPLS pilot, a Consultant Physician provided virtual ward rounds and advisory sessions. 100\% of staff involved reported the service was beneficial for their clinical practice and patient outcomes.

Conclusion. Taking these findings and input from colleagues within AWP and nationally, we created a comprehensive strategic overview on how AWP can deliver high quality physical health care, detailing improvements to make across 5 key domains: Inpatient, Community, Workforce, Education and Information Technology (IT).

Presently, we are working with Clinical Commissioning Groups developing protocols clarifying roles and responsibilities across primary and secondary providers. We are standardising communication between AWP and primary care and expanding links with specialist secondary services (e.g. endocrinology and cardiology). We formed the BRIGHT (Better Recording of Information for Governance and Healthcare in the Trust) project workgroup alongside IT to build safer and more effective records systems.

Medium term recommendations include employing a full-time MPLS Consultant Physician, in addition to 'Physical Health and Wellbeing Workers' in all localities, Advanced Nurse Practitioners (working within structured physical care systems) and more allied health professionals (dieticians, speech therapists and physiotherapists).

In the long term, the new Physical Health, IT and QI working groups will maintain development of these proposals, improve training and supervision for clinicians, and achieve healthcare parity for patients across localities.

\section{The impact of COVID-19 on an inpatient mother and baby unit: a service evaluation}

Joanna Cranshaw*, Gertrude Seneviratne, Ranga Rao, Julia Ogunmuyiwa, Rebecca McMillin, Chukwuma Ntephe and Victoria Dain

South London and Maudsley NHS Foundation Trust

${ }^{\star}$ Corresponding author.

doi: $10.1192 /$ bjo.2021.833

Aims. Unique challenges have been faced by women in the perinatal period during the COVID-19 pandemic and the impact of this is compounded for women suffering from mental illness. This service evaluation looked at different aspects of the treatment pathway on a specialist inpatient psychiatric Mother and Baby Unit during the pandemic to identify what changes occurred.

Method. Data were collected for all admissions to the unit between January 2019 and October 2020, with the beginning of the pandemic being defined as on or after 1st March 2020. Information was collected retrospectively from electronic clinical notes on ethnicity, length of stay, diagnosis, mental health act use and restrictive practice, medication, psychology, occupational therapy and social services involvement.

Result. There were 114 admissions to the MBU during the study period. 4 were parenting assessments rather than acute psychiatric admissions and were excluded from the analysis, giving a sample of 110 women. 58\% (62/110) were classed as "pre-pandemic" and $43.6 \%$ (48/110) were "during pandemic". 95.45\% (105/110) of women were postpartum $4.55 \%$ (5/110) were pregnant. Mean length of stay was shorter during the pandemic at 44 days, compared to 61 pre-pandemic. There was greater use of the mental health act during the pandemic: only $43.75 \%$ of patients were informal throughout admission, compared to $70.97 \%$ prepandemic. Mean duration of detention was shorter at 25 days (32 pre-pandemic). Psychotic illness made up a greater proportion of diagnoses during the pandemic: $56 \%$ (27/48) compared to $44 \%(27 / 62)$ pre-pandemic. The next most common diagnostic group was mood and anxiety disorders, which made up 29\% (14/ 48 ) of diagnoses during the pandemic, but $43 \%$ (27/62) prepandemic. Outcomes as measured using the Health of the Nation Outcome Scale showed a mean improvement between admission and discharge of 6.65, compared to 5.15 pre-pandemic. HONOS scores were higher on admission during the pandemic (12.83, vs 10.88), suggesting a higher level of acuity.

Conclusion. During the COVID-19 pandemic on this Mother and Baby Unit, length of stay was shorter, a greater proportion of patients were detained under the mental health act (although length of detention was shorter) and psychotic illness was more prevalent. This study demonstrates that there were differences in this perinatal inpatient population during the pandemic and this may be a reflection on the wider impact of COVID-19 on perinatal mental health.

\section{Audit of shared-care lithium monitoring in a large rural GP practice}

Thomas Cranshaw

Cumbria, Northumberland Tyne and Wear Foundation Trust

doi: 10.1192/bjo.2021.834

Aims. To compare monitoring of lithium treatment with shared care lithium monitoring agreements in a large rural GP practice. Background. A 'near miss' event with a patient with drug induced long QT syndrome highlighted a need for an audit of lithium monitoring at a large rural GP practice.

The practice had entered into shared-care monitoring agreements with the local mental health care trust. Under these agreements, responsibility for physical monitoring of lithium treatment was assumed by the practice.

Method. Using audit functions built into the IT system, all patients at the practice who were currently prescribed lithiumcontaining medications were identified $(n=28)$. Individual monitoring standards were determined for each patient based on the shared care agreement. These varied depending on age and comorbidity. Monitoring data obtained from medical records was compared against the individualised monitoring requirement. Result. The key finding was that $26 \%$ of patients for whom annual ECGs were indicated according to the shared care agreement had received an ECG in the past year. $78 \%$ of patients had a lithium level recorded in the previous 3 months. $81 \%$ of patients had a renal function test within their monitoring requirements. 52\% of patients had lipid measurement in the previous year.

Conclusion. There is a great degree of heterogeneity in the extent to which shared care monitoring agreements are achieved. It is noted that those standards to which a Quality Outcome Framework incentive applied had a greater chance of being met. 
Worryingly, the QOF statements relating to lithium treatment have now been retired as of April 2019. It is suggested that psychiatrists are aware of the challenges primary care faces when monitoring lithium treatment.

\section{Consultation liaison to support efficient delivery of mental health care}

Emma Davies* and Mihaela Bucur

Sussex Partnership NHS Foundation Trust

${ }^{\star}$ Corresponding author.

doi: 10.1192/bjo.2021.835

Aims. To study the impact of collaborative working, via consultation liaison, between Mental Health Liaison Practitioners (MHLPs) and Doctors within a secondary care mental health service. We hypothesise that this model of working may avoid unnecessary clinic appointments and waiting times, whilst providing patients with more efficient treatment.

Background. Mental health services are stretched, understaffed and under-resourced. It is estimated that $75 \%$ of people with mental health problems in England may not get access to the treatment they need. We therefore need efficient and innovative ways for people who seek help to receive support. Good practice consultation liaison involves face to face contact between clinicians; treatment can be delivered by supporting primary care whilst reducing the burden of secondary care mental health services.

Method. Regular 30-minute sessions within an Assessment and Treatment Service, between MHLPs and Doctors, at both Consultant and Trainee level, were coordinated. Patients assessed by MHLPs were discussed by opening a dialogue whereby further management was discussed across a multi-professional team. A record was created of all patients discussed and the outcome.

Result. Number of MHLP/Doctor sessions: 10 across a six-month period.

Number of patients discussed: 17.

Medication advice provided for 16 patients. One patient required a referral for a clinic appointment.

For several patients, integrated working procured alternative care pathways and resources to be considered, to incorporate into individual treatment plans.

Conclusion. Regular consultation liaison with MHLPs and Doctors is a model of working across the interface between primary care and specialist mental health services. It may provide patients with more efficient care, whilst avoiding unnecessary waiting times for clinic appointments. The consultation liaison working supported the development of an educative relationship between clinicians, with interprofessional learning. This is an example of an integrated and collaborative care model, whereby multi-professional working can provide efficient and effective treatment, whilst the support for the patient can remain in the primary care setting.

\section{Physical health monitoring in antipsychotic depot clinic}

Rebecca Davies ${ }^{1 \star}$, Anu Priya ${ }^{1}$, Hardev Bhogal ${ }^{1}$, Adesola Omodara ${ }^{1}$, George Davies ${ }^{2}$ and Shweta Mittal ${ }^{1}$

${ }^{1}$ Nottinghamshire Healthcare NHS Foundation Trust and

${ }^{2}$ Nottingham Medical University

${ }^{\star}$ Corresponding author.

doi: 10.1192/bjo.2021.836
Aims. A service evaluation project to look at if annual bloods, ECG, physical examination, and medical review was completed within the last year for patients attending anti-psychotic depot clinic at Bassetlaw mental health services in Nottinghamshire HealthCare NHS Foundation Trust.

Method. Electronic notes were examined in October 2020 for 25 patients who attend anti-psychotic depot clinic to ascertain if medical review and physical examination had been completed along with annual bloods and ECG.

Result. Out of 25 patients attending depot clinic in 2020 at Bassetlaw Hospital, 21 had all their blood tests done, 1 patient had refused bloods and 2 patients did not have blood tests done. ECG was completed for 3 patients at Bassetlaw hospital and 8 patients had it requested from primary care with 2 patients refusing to have ECG done. For 12 patients there was no evidence of ECG being requested or completed. 8 patients had physical examination completed and rest 17 patients did not have the physical examination completed including due to refusal. Out of 25 , only 14 patients had a medical review conducted.

Conclusion. Patients who attend depot clinic may have an allocated community psychiatric nurse $(\mathrm{CPN})$ or get reviewed by medics in outpatient clinics and would usually have their blood tests, physical health examination and ECGs requested and monitored by them. Patients who do not have any allocated CPN or medic tend to miss out on blood tests and ECG. General Practitioners are expected to complete physical health checks for patients who do not have CPN or regular outpatient review. The results of these investigations may not always be received in depot clinic, hence there is no documentation on electronic RIO system. When these patients disengage from the depot clinic, it is often very difficult to track them. As a follow-up from this service evaluation, all depot clinic patients will be allocated a key worker/CPN. This will ensure that they have a responsible person to facilitate annual checks. This will be reviewed in a years' time to evaluate the effectiveness of this intervention.

\section{Re-evaluating trainee experience of involvement in serious incidents - has anything changed?}

Glori-Louise de Bernier ${ }^{\star}$, Alice Debelle, Marilia Calcia and Lauren Waterman

South London and Maudsley NHS Foundation Trust ${ }^{\star}$ Corresponding author.

\section{doi: 10.1192/bjo.2021.837}

Aims. To complete an audit cycle to investigate: trainees' experiences of SI involvement since 2017, perceptions of current support systems and trust facilitation of learning from SIs and the impact of the interventions implemented following the 2017 survey.

Background. In 2017, data were collected from trainees working in psychiatry within two London trusts to examine the nature of their involvement in serious incidents (SIs), their experience of the process following an SI and their knowledge of the support systems available to them. Due to concerning results from this, several interventions were put in place in accordance with trainees' suggestions.

Method. Cross-sectional surveys were e-mailed to trainees of all grades in July 2019, including GP and foundation doctors, working within two mental health trusts. These built upon the 2017 surveys, additionally enquiring about demographic information and the personal and training consequences of SIs on trainees.

Result. 61 (15\% of all trainees) returned the survey with 41 (67\%) respondents unable to recall any SI related teaching during induction and $47(77 \%)$ not having received a written guidance document on SI procedures. 